\title{
MRI imaging of vein of Galen malformations at Steve Biko Academic Hospital: A mini case series
}

\author{
Liesel Davel, MB ChB, DA (SA), MMed Rad (D) \\ Janet Smal, MB ChB, MMed Rad (D) \\ Z I Lockhat, FCRad (D) \\ Department of Radiology, Steve Biko Academic Hospital, Pretoria
}

Corresponding author: L Davel (liesel-davel@hotmail.com)

\section{Introduction}

Vein of Galen aneurysmal malformation (VOGM) is an arterio-venous fistula involving aneurysmal dilatation of the median prosencephalic vein of Markowski. Dilated arteries drain into a large midline venous pouch in the region of the quadrigeminal cistern. It is a rare condition and is demonstrated in less than $1 \%$ of cerebral vascular malformations at any age and up to $30 \%$ of paediatric vascular malformations. Associated clinical findings may include cardiomegaly, widened mediastinum, pulmonary oedema and cyanosis. ${ }^{1-3}$ Heart failure and cyanosis in infants almost always results from congenital heart disease, but systemic arteriovenous malformations are a rare cause for such a presentation and the diagnosis is often missed or delayed. ${ }^{4}$ The following is a discussion and presentation of MRI images of 4 patients with this rare condition who presented at Steve Biko Academic Hospital within a 2-year period.

\section{Case 1}

An 8-month old baby presented to the Paediatric Department with increasing head circumference, above the 97 th percentile, delayed motor milestones with an inability to sit, and neuro-developmental delay. On clinical examination, an audible cranial bruit and cardiac ejection systolic murmur were found. The child was in cardiac failure. MRI examination confirmed a vein of Galen malformation (Fig. 1). A well-defined midline pouch was demonstrated in the posterior fossa, communicating with the left posterior cerebral artery. The circle of Willis demonstrated large congested arteries owing to the arteriovenous fistula. Smaller feeding arteries were noted adjacent to the vein of Galen. A ventriculo-peritoneal shunt was inserted, and the child's neurological condition improved. This was followed by endovascular intervention. The child is being followed up and is currently doing well.

\section{Case 2}

A 4-week-old baby was referred from a peripheral clinic with clinical signs and symptoms of cardiac failure and an incidental cranial bruit. This prompted further evaluation to exclude an associated intracranial shunt. MRI confirmed a vein of Galen malformation (Fig. 2) with several dilated intracranial arteries (branches of the anterior cerebral artery) communicating with a large midline venous pouch. This in turn drained via falcine veins into the large transverse and sigmoid sinuses. An unusual associated finding of large bilateral chronic subdural effusions was demonstrated, with significant cortical atrophy. Cystic encephalomalacia was present, mainly on the right. The child subsequently died before any interventional procedures could be performed.

\section{Case 3}

A 2-year-old child with neuro-developmental delay presented acutely with convulsions and opisthotonus. The child had been unable to stand or walk prior to the seizure. The child's general condition was good but, on examination, a left hemiparesis was present. Initially, a CT scan was performed and revealed a left temporal lobe intra cranial bleed and acute hydrocephalus. MRI (Fig. 3) confirmed a left temporal lobe arterio-venous malformation with secondary intracerebral bleeding as well as a well-defined, enlarged venous pouch in keeping with a vein of Galen malformation.

\section{Case 4}

This case was of a more typical vein of Galen malformation with an atypical clinical presentation. The child presented at 2 months of age with the sole clinical problem of hypotonia. The only significant history at that stage was that the child had been born with a low Apgar score. Skull sonar was the initial examination and revealed dilated lateral and 3 rd ventricles, which prompted a request for a CT scan that was initially diagnosed as hydrocephalus secondary to a pituitary gland cyst. To confirm the diagnosis, an MRI was performed, which revealed a large posterior midline venous pouch with arteries draining into the pouch; the diagnosis of a vein of Galen malformation was made (Fig. 4).

\section{Discussion}

A vein of Galen aneurysm is a rare condition, demonstrated in less than $1 \%$ of cerebral vascular malformations at any age and up to $30 \%$ of paediatric vascular malformations. The vein of Galen (formed from the median vein of the prosencephalon) is formed by the union of the two internal cerebral veins and goes on to drain into the straight sinus. An arterio-venous malformation (AVM) occurs when the vein of Galen has an arterial input from one or more major intracranial arteries, either directly or via an interposed angiomatous malformation. ${ }^{4}$

\section{Clinical presentation}

Neonates with vein of Galen malformations characteristically have multiple fistulae. Up to $25 \%$ of their cardiac output passes through the fistulas, causing high-output congestive cardiac failure. Depending on the size of the shunt, adequacy of venous drainage, complexity of arterial 
supply and the host response, the cardiac manifestations can range from asymptomatic cardiomegaly to severe cardiac failure that is refractory to medical management. Cyanosis may be seen in these patients, and the presentation may be mistaken for congenital cyanotic heart disease. ${ }^{4,5}$

Older children and adults usually have low-flow fistulae. These patients usually present with headache and seizures. A small number of patients may also present with developmental delay, focal neurological deficits, proptosis and epistaxis. Subarachnoid haemorrhage and intracerebral haemorrhage can occur in this age group, owing to re-routing of blood into the pial veins. ${ }^{4,5}$

In cases 1 and 4 , the children presented with less acute clinical symptoms. Neuro-developmental delay, increased head circumference and hypotonia were some of the presenting features, and symptoms of cardiac failure were more subtle. These two cases also had more typical MRI features suspected in vein of Galen malformations.

In case 1, the large left posterior cerebral artery feeder is less typical. Arterial feeders are usually one of two types. The choroidal type occurs when arterial feeders originate from the pericallosal, choroidal or thalamoperforaters. If the arterial feeders originate from the collicular or posterior choroidal arteries, they are classified as the mural type. ${ }^{1}$

In case 2 , the baby presented with typical clinical findings of severe cardiac failure. The associated MRI findings were less typical. Bilateral subdural effusion, cerebral atrophy and cystic encephalomalacia are most likely attributed to either hypoxic ischaemic incident at birth, vascular steal or chronic venous hypertension.

In case 3 , the child was already 2 years old when he presented with convulsions and opisthotonus. Before the seizure, there was a background history of neuro-developmental delay. The atypical imaging feature in this patient was the finding of a second arteriovenous malformation in the left temporal lobe. This is the malformation that bled and caused the acute neurological impairment.

In all 4 cases, there was some degree of hydrocephalus present. Hydrocephalus in this condition is due to decreased resorption of cerebrospinal fluid secondary to chronic venous hypertension or tectal compression. ${ }^{1}$

In 2 of the 4 cases, the babies presented with cardiac failure. Developmental delay, hydrocephalus and seizures were also presenting symptoms.

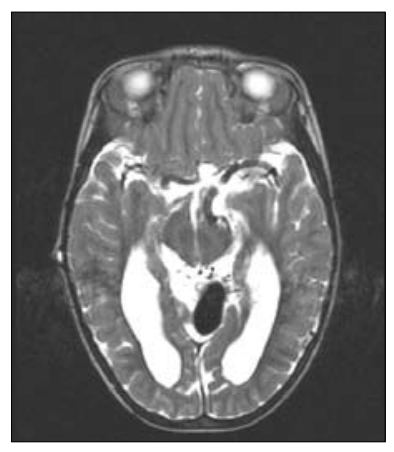

Fig. 1a. T2-weighted TSE axial view.

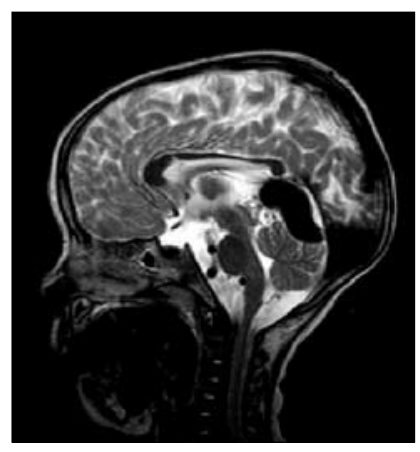

Fig. 1b. T2-weighted TSE sagittal view.

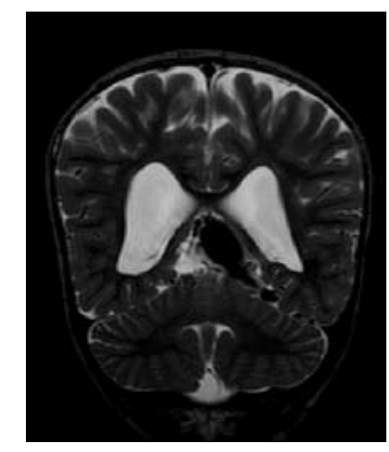

Fig. 1c. T2-weighted TSE coronal view.

\section{Imaging}

Radiography. Plain radiography of the skull contributes little to the diagnosis of VOGMs beyond the demonstration of a rim of calcification within the wall of the aneurysmal sac. Chest radiographs may reveal features of congestive heart failure.

Ultrasound. Antenatal ultrasound scans demonstrate the venous sac as a sonolucent mass located posterior to the third ventricle. Ultrasonic demonstration of pulsatile flow within it helps in differentiating VOGMs from other midline cystic lesions. Associated venous anomalies can often be visualized. Evidence of hydrocephalus and cardiac dysfunction can also be obtained on antenatal ultrasonography. ${ }^{4,5}$

Computed tomography (CT). Contrast-enhanced axial CT scan of the brain usually demonstrates a well-defined, multilobulated, intensely enhancing lesion, located within the cistern of velum interpositum. Dilatation of the ventricular system, periventricular white matter hypodensities, as well as diffuse cerebral atrophy are the commonly associated findings. ${ }^{4,5}$

Magnetic resonance imaging (MRI). MRI is gaining popularity as the modality of choice for initial assessment of VOGMs. It can demonstrate the location of fistula, presence of any nidus, the arterial components, the venous sac and the status of venous drainage. Thrombosis of the venous sac is also depicted well on MRI. MR angiography is being increasingly used as a non-invasive alternative to diagnostic angiographic studies in the initial evaluation of these lesions. ${ }^{4,5}$

Angiography. Angiography remains the gold standard for the evaluation of VOGMs. It scores over non-invasive modalities such as CT angiography and MR angiography in demonstrating small feeders supplying the fistula, as well as the dynamic aspects of the venous drainage of the normal brain, and haemodynamic relationships with the venous drainage of the arteriovenous shunt. ${ }^{4,5}$

\section{Management}

Despite technological advances in micro-neurosurgery, complete elimination of the lesion by surgery is rarely achieved. ${ }^{4,5}$ Though these lesions are extremely uncommon, they are of special interest to the interventional neuroradiologist because endovascular therapy has proved itself to be an effective, and often the only safe, therapeutic modality available to treat these patients. ${ }^{4,5}$ The timing of endovascular

Fig. 1. A midline dilated venous pouch with characteristic flow void is seen on axial (Fig. 1a) and sagittal (Fig 1b) images. There are multiple small arterial feeders in the midline with a prominent left posterior cerebral artery communicating with the venous pouch (Fig. 1c). This direct posterior cerebral artery shunt is causing dilatation of the circle of Willis (Figs $1 a$ and $1 d$ ). 


\section{PICTORIAL ESSAY}

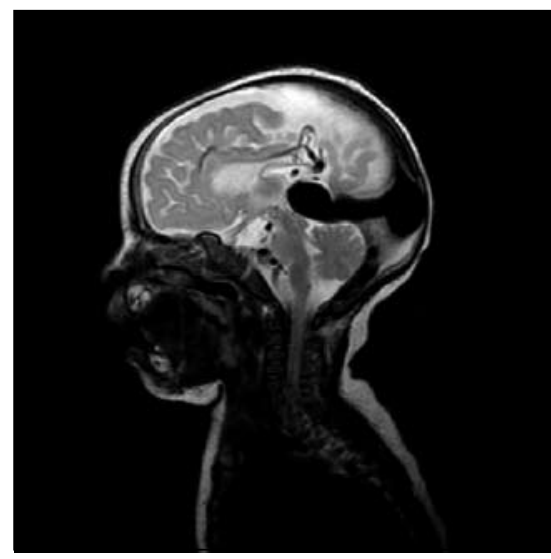

Fig. 2a. T2-weighted TSE sagittal image.

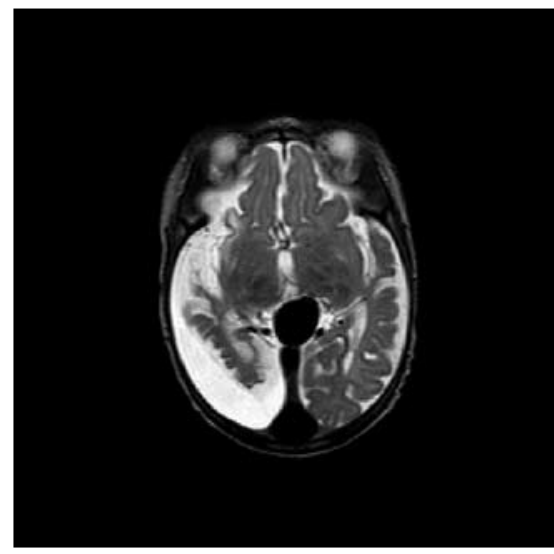

Fig. 2b. T2-weighted TSE axial image.

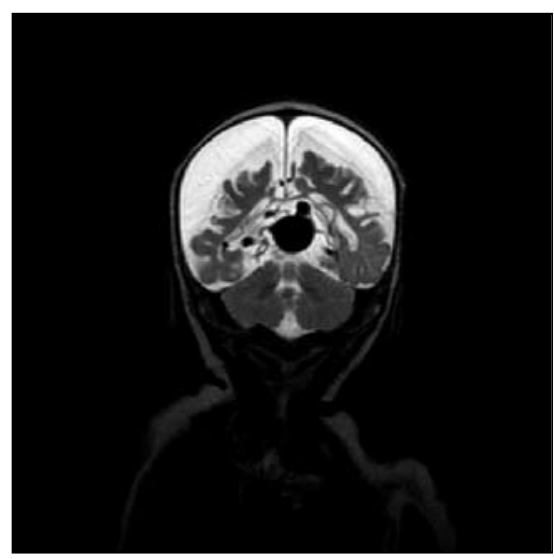

Fig. 2c. T2-weighted TSE coronal image.

Fig. 2. Multiple arterial feeders drain into a large dilated midline venous pouch (Figs $2 a-c)$. The large midline venous pouch in turn drains via falcine veins into the large transverse and sigmoid sinuses. Large bilateral chronic subdural effusions are demonstrated, with significant cortical atrophy (Fig. 2c).

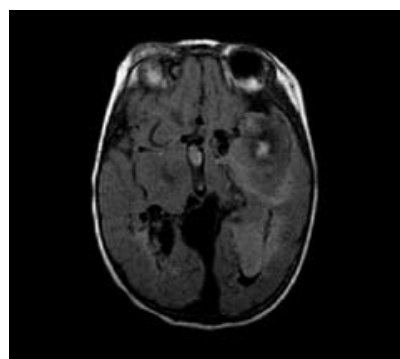

Fig. 3a. T1-weighted TSE axial Fig. 3b. T1-weighted postimage.

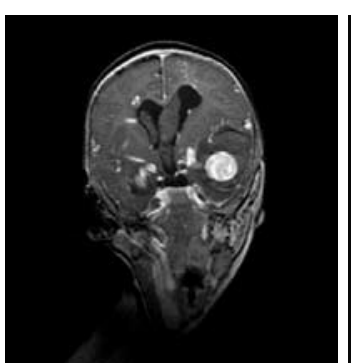

gadolinium coronal image.

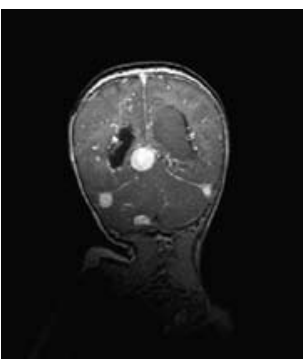

Figure 3c. T1-weighted Fig. 3d. T1-weighted post- Fig. 3e.T1-weighted post-

post-gadolinium coronal gadolinium sagittal image. gadolinium axial image. image.
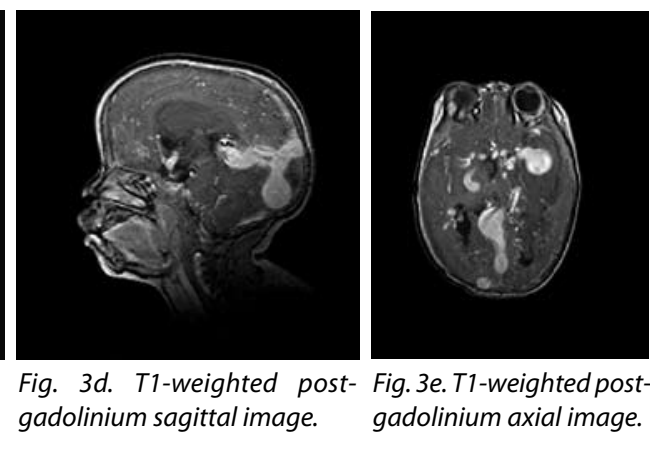

Fig. 3. An atypical case demonstrating an arteriovenous malformation in the left temporal lobe complicated by haemorrhage (Figure $3 a$ and $3 b$ ) as well as the enhancing midline venous pouch of the vein of Galen malformation (Figs $3 c-e$ ).

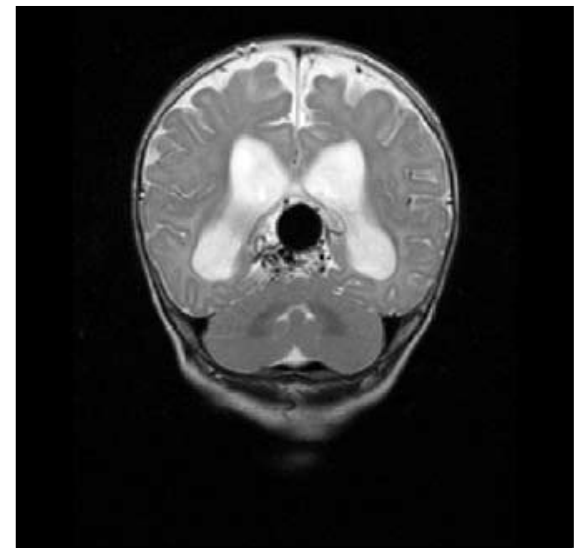

Fig. 4a. T2-weighted TSE coronal image.

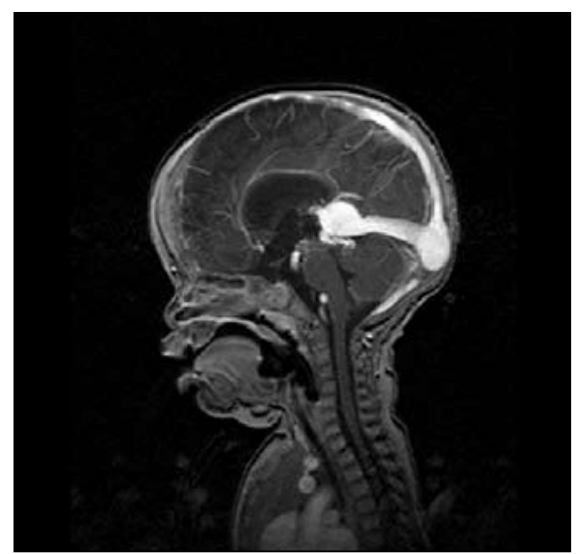

Fig. 4b. T1-weighted post-gadolinium sagittal image.

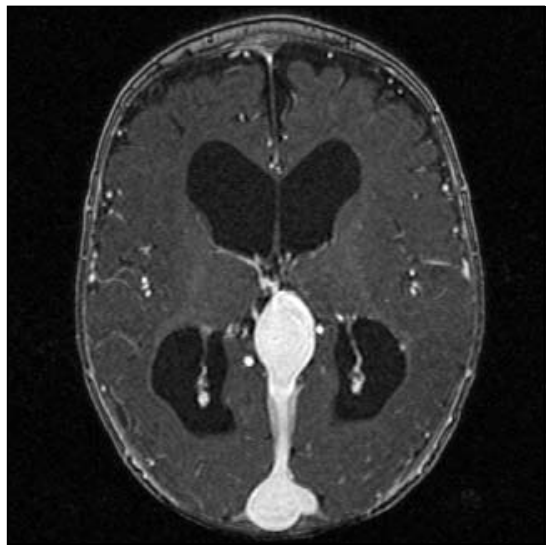

Fig. 4c. MR angiogram axial image.

Fig. 4. A typical case of a large posterior midline venous pouch (Figs $4 a$ and 4c) with multiple arterial feeding vessels draining into the midline pouch. Dilated lateral ventricles are due to non-obstructive communicating hydrocephalus. Enhancement of the venous pouch is demonstrated post-gadolinium (Fig. $4 b$ ) and by MR angiography (Fig. 4c).

management is determined by the clinical presentation. Congestive cardiac failure in a neonate that is refractory to medical treatment is an indication for emergency embolisation. The goal of therapy in such patients would be to arrest the congestive cardiac failure rather than to achieve complete obliteration of the shunt. In such children, it may be acceptable to perform partial embolisation to reduce the arteriovenous shunt and facilitate normal systemic and neurological development, even with the presence of a residual shunt. The procedure can be performed in a staged manner to minimise complications. ${ }^{4,5}$
1. Moore KR, Barkovich AJ, Grant E, et al. Diagnostic Imaging: Pediatric Neuroradiology. 1st ed. Salt Lake City: Amirsys, 2007: 1:7:26-29.

2. Barkovich AJ. Paediatric Neuroimaging. 4th ed. Philadelphia: Lippincott Williams \& Wilkins, 2005:875884.

3. Atlas SW. Magnetic Resonance Imaging of the Brain and Spine. 3rd ed. Philadelphia: Lippincott Williams \& Wilkins, 2002:337-339

4. Kothari SS, Naik N, Juneja R, Saxena A. Aneurysm of vein of Galen in the neonates: Report of four cases. Indian Heart Journal 2001;53:499-502

5. Gupta AK, Varma DR. Vein of Galen malformations: Review. Neurol India 2004;52:43-53. 